\title{
Rosiglitazone alleviates injury in rats with adenine-induced chronic kidney disease
}

\author{
YING HUANG, YAN LEI, ZHIHUA ZHENG, XIAOHUA WANG, \\ MINGLIANG HU, RONGJUN LIU and XUEQING YU

\begin{abstract}
Department of Nephrology, The First Affiliated Hospital, Sun Yat-Sen University, Key Laboratory of Nephrology, Ministry of Health, Guangzhou, Guangdong 510080, P.R. China
\end{abstract}

Received May 29, 2013; Accepted September 24, 2013

DOI: $10.3892 / \mathrm{mmr} .2013 .1715$

\begin{abstract}
Rosiglitazone (ROG) has been shown to exert beneficial effects on glycemic control and renal protection. Circulatory fibroblast growth factor-23 (FGF-23) is a novel independent risk factor for chronic kidney disease (CKD) progression. The current study focused on how ROG impacts on injury of the kidney, and whether FGF-23 is involved in the process. A total of 24 male Sprague Dawley rats, weighing 250-280 g, were divided into four groups ( $\mathrm{n}=6$ per group): i) Normal; ii) ROG controls, treated with ROG (10 mg/kg/day); iii) CKD models, treated with adenine (200 mg/kg/day); and iv) ROG treatment, treated with ROG $(10 \mathrm{mg} / \mathrm{kg} /$ day $)$ and adenine $(200 \mathrm{mg} / \mathrm{kg} /$ day $)$. The rats were sacrificed after four weeks, and serum, urine and kidney tissues were collected. The data revealed that the serum levels of inorganic phosphate (Pi), intact parathyroid hormone (iPTH) and FGF-23 were significantly higher in the CKD models compared with those in the normal group $(\mathrm{P}<0.01)$, while ROG significantly reduced the serum levels of $\mathrm{Pi}$, iPTH and FGF-23 $(\mathrm{P}<0.01)$. The ratio of protein/creatine $(\mathrm{Cr})$ in the urine and the serum levels of $\mathrm{Cr}$, blood urea nitrogen and uric acid were significantly increased in the CKD models compared with the normal group $(\mathrm{P}<0.01)$, however, ROG significantly reduced these parameters $(\mathrm{P}<0.05)$. Furthermore, ROG significantly alleviated the tubule interstitial damage index of the CKD models in comparison with that of the controls. These results indicated that ROG mitigates the lesions of chronic kidney disease induced by adenine, and that the system of Pi-PTH-FGF-23 may contribute to this process. The possible
\end{abstract}

Correspondence to: Professor Zhihua Zheng, Department of Nephrology, The First Affiliated Hospital, Sun Yat-Sen University, Key Laboratory of Nephrology, Ministry of Health, 58 Zhongshan Road II, Guangzhou, Guangdong 510080, P.R. China

E-mail: zhihuazheng@126.com

Key words: rosiglitazone, chronic kidney disease, proteinuria, renal function, phosphate-parathyroid hormone-fibroblast growth factor-23 system mechanisms underlying this process require confirmation in future studies.

\section{Introduction}

Chronic kidney disease (CKD) is currently considered to be a predominant public health burden and a major cause of morbidity and mortality for diabetic and hypertensive patients worldwide. Recent clinical studies have revealed that persistent proteinuria and serum FGF-23 levels are powerful risk factors for the progression of end-stage renal disease (1-4).

Fibroblast growth factor-23 (FGF-23) is a newly identified member of the FGF family. FGF-23 is secreted from the bone and acts on the kidney to promote urinary inorganic phosphate (Pi) excretion and to suppress vitamin D synthesis, thereby maintaining Pi and calcium homeostasis $(5,6)$. An increasing number of studies have confirmed that the circulatory levels of FGF-23, PTH and Pi are independent risk factors for CKD progression, and are closely correlated with vascular calcification and secondary hyperparathyroidism (7-9).

Proteinuria is a powerful and independent risk factor for kidney disease, cardiovascular disease and all-cause mortality (10). Thus, the reduction in proteinuria invariably translates into protection from a decline in renal function in order to improve the clinical outcome. All studies are committed to reduce proteinuria, improve renal function and extenuate complications in CKD patients (8-10).

Thiazolidinediones (TZDs) are ligands known to bind and activate the nuclear peroxisome proliferator-activated receptor- $\gamma$ (PPAR- $\gamma$ ). TZDs are used to treat diabetes mellitus, and are known to exert their glucose-lowering effects by reducing insulin resistance through the stimulation of PPAR- $\gamma$ nuclear receptor. In addition to improving glycemic control, TZDs have been demonstrated to exert beneficial effects on renal and vascular protection (11-15). In the present study, the effect of rosiglitazone (ROG) on proteinuria and renal function in CKD models induced by adenine was analyzed, and the contribution of the Pi-PTH-FGF-23 system was investigated.

\section{Materials and methods}

Animal study protocols. This study was conducted according to the guidelines of the National Institute of Health on the care 
and use of laboratory animals. The project was approved by the Institutional Animal Care and Use Committee of Sun Yat-Sen University. All rats were housed individually and were provided with normal laboratory chow and tap water ad libitum throughout the study. The animals were handled daily to minimize handling stress. Male SD rats (weight, 250-280 g) were obtained from the Animal Center of Sun Yat-Sen University (Guangzhou, China). The SD rats were divided randomly into four groups ( $n=6$ per group): i) Normal group, intragastric administration of saline consistently for four weeks; ii) ROG controls, intragastric administration of rosiglitazone (GlaxoSmithKline plc., Tianjin, China) $10 \mathrm{mg} / \mathrm{kg} /$ day consistently for four weeks; iii) CKD model group, intragastric administration of $200 \mathrm{mg} / \mathrm{kg} /$ day adenine (Sigma-Aldrich, St. Louis, MO, USA) consistently for four weeks; and iv) ROG treatment group, intragastric administration of ROG (10 mg/kg/day) and adenine (200 mg/kg/day) consistently for four weeks. All rats were sacrificed, and the kidney tissues and blood samples were collected at the end of four weeks. Urine samples of 24-h were collected on the day prior to the rats being sacrificed.

Biochemistry assay. The blood and urine samples from the rats were centrifuged at $1,120 \mathrm{x}$ g for $10 \mathrm{~min}$. The levels of serum creatine $(\mathrm{Cr})$, blood urea nitrogen $(\mathrm{BUN})$, uric acid (UA), calcium, phosphorus, intact parathyroid hormone (iPTH), alkaline phosphatase (ALP) and the 24 -h protein/Cr ratio of the urine were tested by an autoanalyzer (Abbott, Chicago, IL, USA) in duplicate.

Enzyme-linked immunosorbent assay (ELISA). The serum levels of FGF-23 were measured by ELISA (FGF-23 ELISA kit; Immutopics, San Clemente, CA, USA) according the manufacturer's instructions.

Hematoxylin and eosin (HE) staining and pathological analysis. The kidneys were fixed in $4 \%$ paraformaldehyde overnight and embedded with paraffin for HE staining. Semi-quantitative histological scoring was performed by a pathologist. The scoring of the tubular interstitial damage index (TIDI) used the following criteria: The tubular atrophy and interstitial infiltrates were scored as absent, 0 ; involving $<25 \%$ of the biopsy area, 1; involving 26 to $50 \%$ of the biopsy area, 2 ; and involving $>50 \%$ of the biopsy area, 3 . The presence of proteinaceous casts was scored as absent, 0 ; isolated, 1 ; present in $\leq 10 \%$ of tubules, 2 ; and present in $>10 \%$ of tubules, 3 . Tubular dilation was scored as absent, 0; mild, 1; moderate, 2, when associated with flattening of the tubular epithelium; or severe, 3 . Tubular dilation was scored as severe, 3 , when the diameter exceeded that of the glomeruli; these tubules were often filled with proteinaceous casts.

Statistical analysis. The statistical analysis was conducted using analysis of variance (SPSS 13.0 software: SPSS, Inc., Chicago, IL, USA). $\mathrm{P}<0.05$ was used to indicate a statistically significant difference. The results are expressed as the mean $\pm \mathrm{SE}$.

\section{Results}

Effect of ROG on the Pi-PTH-FGF-23 system. The serum levels of FGF-23 were significantly higher in the
$\mathbf{A}$

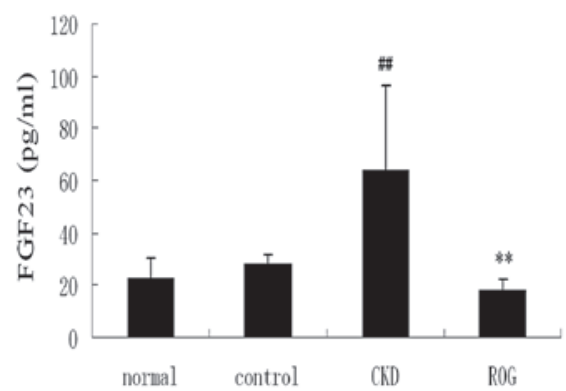

B

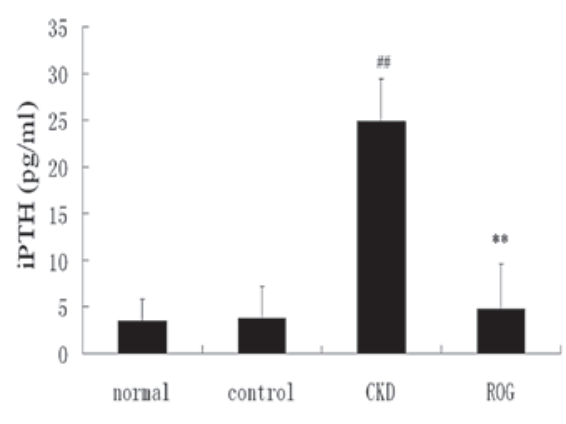

C

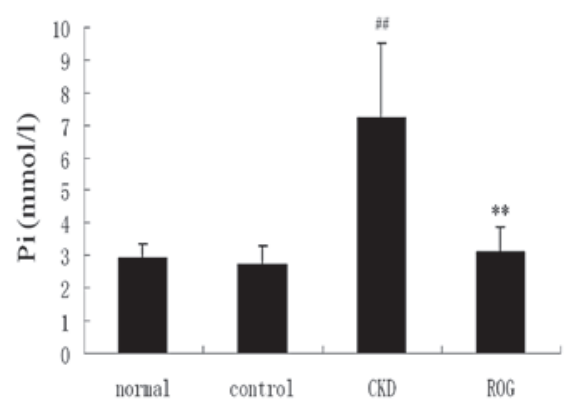

Figure 1. (A) Effects of rosiglitazone (ROG) on serum FGF-23 levels among four groups. (B) Effects of ROG on serum PTH levels among four groups. (C) Effects of ROG on serum phosphate (Pi) levels among four groups. ${ }^{\#} \mathrm{P}<0.05$ and ${ }^{\# \#} \mathrm{P}<0.01$ vs. control; ${ }^{*} \mathrm{P}<0.05$ and ${ }^{* *} \mathrm{P}<0.01$ vs. model. $\mathrm{PTH}$, parathyroid hormone; CKD, chronic kidney disease.

CKD model group compared with those of the normal group $(64.3 \pm 13.2$ versus $23.2 \pm 7.2 \mathrm{pg} / \mathrm{ml} ; \mathrm{P}<0.01)$. The value of FGF-23 was significantly decreased in the ROG treatment group compared with the CKD model group (17.9 \pm 4.5 versus $64.3 \pm 13.2 \mathrm{pg} / \mathrm{ml} ; \mathrm{P}<0.01)$. The serum levels of iPTH and Pi were also significantly higher in the CKD model compared with the normal group $(\mathrm{P}<0.01)$, but significantly reduced following treatment with $\mathrm{ROG}$ in comparison with the CKD model $(\mathrm{P}<0.01$; Fig. 1A-C). However, there were no significant differences in serum calcium and ALP levels among the four groups.

Effect of ROG on proteinuria and renal function. The ratio of protein/Cr of the urine was significantly increased in the CKD models compared with that in the normal group $(6,391.4 \pm 1,923.0$ versus $1,526.1 \pm 719.5 \mathrm{mg} / \mathrm{g} ; \mathrm{P}<0.01)$. However, ROG significantly reduced the proteinuria content compared with the CKD models $(3,469.75 \pm 887.55$ versus 6,391.41 $\pm 1,923.02 \mathrm{mg} / \mathrm{g} ; \mathrm{P}<0.01$ )(Fig. 2A-C). Serum Cr, BUN and UA levels were also significantly increased in the CKD models group compared with the normal group. It is noteworthy that the levels of renal function were significantly improved following treatment with ROG (Fig. 3A-C). 
Table I. Comparison of the TIDI in the various groups of the experiment.

\begin{tabular}{lcccc}
\hline Parameter & Normal controls & ROG controls & CKD models & ROG treatment group \\
\hline TIDI & $0.53 \pm 0.19$ & $0.61 \pm 0.22$ & $9.33 \pm 1.74^{\mathrm{a}}$ & $5.95 \pm 0.89^{\mathrm{a}, \mathrm{b}}$ \\
\hline
\end{tabular}

${ }^{\mathrm{a}} \mathrm{P}<0.01$ vs. control; ${ }^{\mathrm{P}}<0.05$ and vs. model. TIDI, tubular interstitial damage index; ROG, rosiglitazone; CKD, chronic kidney disease.

A

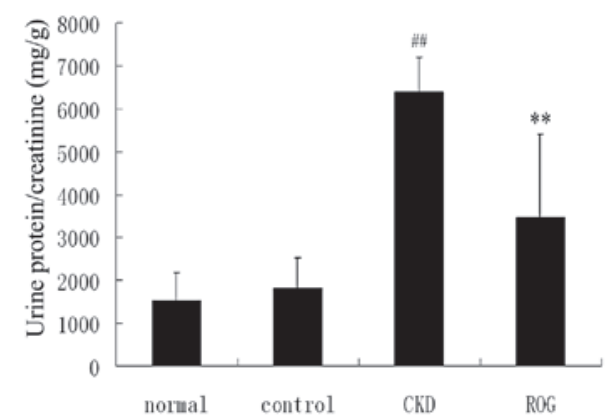

B

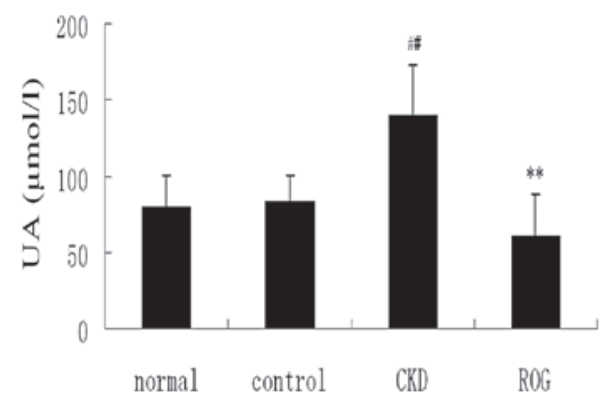

Figure 2. (A) Effects of rosiglitazone (ROG) on the ratio of protein/Cr of the 24-h urine among the four groups. (B) Effects of ROG on the serum uric acid among the four groups. ${ }^{\#} \mathrm{P}<0.05$ and ${ }^{\# \#} \mathrm{P}<0.01$ vs. control; ${ }^{*} \mathrm{P}<0.05$ and ${ }^{* *} \mathrm{P}<0.01$ vs. model. $\mathrm{CKD}$, chronic kidney disease; $\mathrm{Cr}$, creatinine.

A

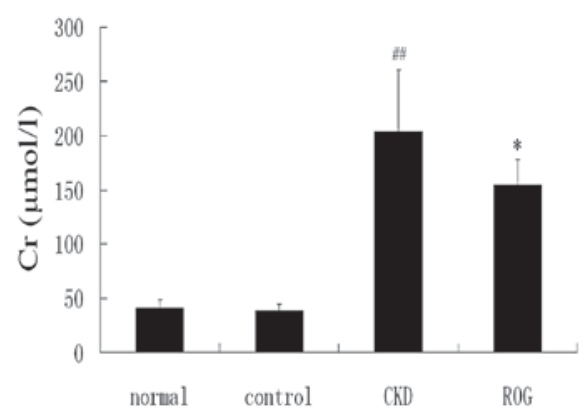

B

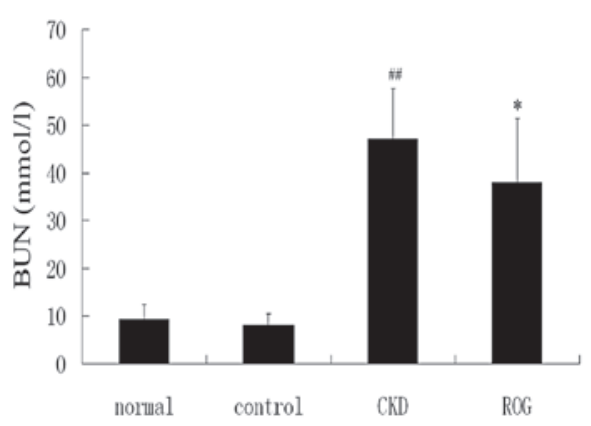

Figure 3. (A) Effects of rosiglitazone (ROG) on serum creatinine (Cr) levels among the four groups. (B) Effects of ROG on serum BUN levels among the four groups. ${ }^{\#} \mathrm{P}<0.05$ and ${ }^{\# \#} \mathrm{P}<0.01$ vs. control; ${ }^{*} \mathrm{P}<0.05$ and ${ }^{* *} \mathrm{P}<0.01$ vs. model. BUN, blood urea nitrogen; $\mathrm{CKD}$, chronic kidney disease.
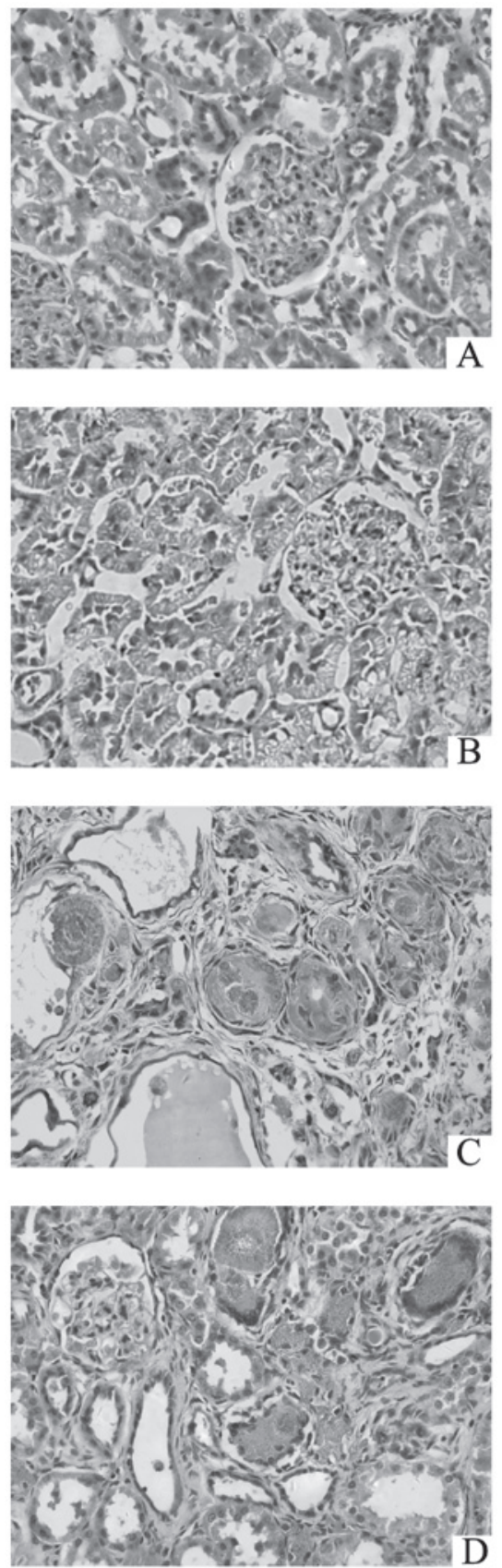

Figure 4. Hematoxylin and eosin staining of the kidney in each group (magnification, $\mathrm{x} 400$ ). (A) Normal; (B) rosiglitazone (ROG) controls; (C) chronic kidney disease models; and (D) ROG treatment.

Renal pathological variation. HE staining demonstrated that the proximal convoluted tubules were swollen, while the distal convoluted tubules and the collecting ducts were expanded with 
considerable protein casts and 2,8-dihydroxyadenine crystals in the lumina (Fig. 4C). Monocytes proliferated around the crystals and created nodules, and inflammatory cells infiltrated into the interstitial tissue (Fig. 4C). However, ROG markedly improved the aforementioned pathological lesions, as shown in Fig. 4D. The TIDI values of the CKD models were significantly increased compared with those of the controls $(\mathrm{P}<0.01)$, while ROG also significantly improved the TIDI ( $<<0.05$, Table I).

\section{Discussion}

In the present study, the CKD rat models were successfully induced with an intragastric administration of adenine, and the serum levels of FGF23, PTH and Pi were confirmed to be consistent with the levels in the lesions of the kidney in this model. Furthermore, this is the first study to demonstrate that ROG was able to attenuate renal damage and metabolic disorders in adenine-induced CKD models. ROG, a PPAR- $\gamma$ agonist, has been indicated to exert cardiovascular and renal protection through the improvement of anti-inflammation, antiproliferation and antioxidative stress (13-18). These data demonstrated that ROG exerts a polyphenic profit on the cardiovascular and renal system. However, the effect of PPAR- $\gamma$ on renal proteinuria and renal function are not yet fully understood.

The present study confirmed the effect of ROG on FGF-23 levels and proteinuria, each a risk factor of CKD, thus providing a novel insight into the renoprotective properties of the drug. The results of this study demonstrated that the serum FGF-23, PTH and $\mathrm{Pi}$ levels were markedly increased in adenine-induced CKD rats, but that ROG was able to effectively attenuate the augmentation. FGF-23 is part of a previously unrecognized hormonal bone-PTH-kidney axis. Mineral and bone metabolism may be viewed as a complex system in which three hormones $(\mathrm{PTH}$, calcitriol and FGF-23) act on three key organs (the kidneys, intestines and bones) to adjust plasma, calcium and phosphorus concentrations (19). Mineral disorders, including an increase in FGF-23 and PTH, and hyperphosphatemia, are independent risk factors in CKD progression and mortality $(3,20,21)$. The present data revealed that ROG reduced the serum levels of FGF-23, PTH and phosphorus in the CKD rats, indicating that ROG may have a beneficial effect on the kidney through the attenuation of mineral disorders in adenine-induced CKD models.

It was also observed that the intervention with ROG decreased the proteinuria and protected the renal function in the adenine-induced CKD models. The present results were consistent with other experimental studies that demonstrated that TZDs are beneficial for renal and cardiovascular protection $(13,18,22,23)$. ROG intervention attenuated hyperuricemia, which is generally acknowledged as an independent risk factor of CKD. Thus, the current study revealed that ROG, a PPAR- $\gamma$ agonist may decrease proteinuria and attenuate metabolic disorders, including mineral disorders and hyperuricemia, in adenine-induced CKD models. Therefore, a novel renoprotective effect of TZDs may be indicated based on the present study. Further studies are required to confirm these findings.

\section{Acknowledgements}

The authors would like to thank Professor Wenfang Chen from the Department of Pathology, The First Affiliated Hospital,
Sun Yat-Sen University. This study was financially supported by the Natural Science Foundation of Guangdong Province, China (grant no. 2012B031800448) and the Foundation of The Educational Ministry (grant no. 2013BSD).

\section{References}

1. Bello AK, Hemmelgarn B, Lloyd A, James MT, Manns BJ, Klarenbach S, et al: Associations among estimated glomerular filtration rate, proteinuria, and adverse cardiovascular outcomes. Clin J Am Soc Nephrol 6: 1418-1426, 2011.

2. Sarnak MJ and Astor BC: Implications of proteinuria: CKD progression and cardiovascular outcomes. Adv Chronic Kidney Dis 18: 258-266, 2011

3. Faul C: Fibroblast growth factor 23 and the heart. Curr Opin Nephrol Hypertens 21: 369-375, 2012.

4. Nakano C, Hamano T, Fujii N, Obi Y, Matsui I, Tomida K, et al: Intact fibroblast growth factor 23 levels predict incident cardiovascular event before but not after the start of dialysis. Bone 50: 1266-1274, 2012.

5. Razzaque MS and Lanske B: The emerging role of the fibroblast growth factor-23-klotho axis in renal regulation of phosphate homeostasis. J Endocrinol 194: 1-10, 2007.

6. Shimada T, Kakitani M, Yamazaki Y, Hasegawa H, TakeuchiY, Fujita T, et al: Targeted ablation of Fgf 23 demonstrates an essential physiological role of FGF23 in phosphate and vitamin D metabolism. J Clin Invest 113: 561-568, 2004.

7. Seiler S, Heine GH and Fliser D: Clinical relevance of FGF-23 in chronic kidney disease. Kidney Int Suppl 76: S34-S42, 2009.

8. El-Abbadi MM, Pai AS, Leaf EM, Yang HY, Bartley BA, Quan KK, et al: Phosphate feeding induces arterial medial calcification in uremic mice: role of serum phosphorus, fibroblast growth factor-23, and osteopontin. Kidney Int 75: 1297-1307, 2009.

9. Gutiérrez OM, Mannstadt M, Isakova T, Rauh-Hain JA, Tamez H, Shah A, et al: Fibroblast growth factor 23 and mortality among patients undergoing hemodialysis. N Engl J Med 359: 584-592, 2008.

10. Sharma SK, Zou H, Togtokh A, Ene-Iordache B, Carminati S, Remuzzi A, et al: Burden of CKD, proteinuria, and cardiovascular risk among Chinese, Mongolian, and Nepalese participants in the International Society of Nephrology screening programs. Am J Kidney Dis 56: 915-927, 2010.

11. Reel B, Guzeloglu M, Bagriyanik A, Atmaca S, Aykut K, Albayrak $G$ and Hazan E: The effects of PPAR- $\gamma$ agonist pioglitazone on renal ischemia/reperfusion injury in rats. J Surg Res 182: 176-184, 2013.

12. Basturk T, Unsal A, Ulas T, Koc Y, Sakaci T, Ahbap E and Borlu F: Effects of rosiglitazone treatment on insulin resistance and TNF-alpha levels in patients with chronic kidney disease: a prospective study. Eur Rev Med Pharmacol Sci 16: 1519-1524, 2012.

13. Setti G, Hayward A, Dessapt C, Barone F, Buckingham R, White $\mathrm{K}$, et al: Peroxisome proliferator-activated receptor- $\gamma$ agonist rosiglitazone prevents albuminuria but not glomerulosclerosis in experimental diabetes. Am J Nephrol 32: 393-402, 2010.

14. Ren L, Liu N, Zhi H, Li Y, Li Y, Tang R, et al: Vasculoprotective effects of rosiglitazone through modulating renin-angiotensin system in vivo and vitro. Cardiovasc Diabetol 10: 10, 2011.

15. Alessi A, França Neto OR, Prim C, Silva RF, Noronha Ld, Brofman PR, et al: Rosiglitazone and vascular injury in hypercholesterolemic rabbits: neointimal formation assessment. Arq Bras Cardiol 95: 283-288, 2010.

16. Nakaya H: Roles of PPARgamma in preventing the development of atherosclerosis in LDL receptor null mice. Nihon Rinsho 68: 229-234, 2010 (In Japanese).

17. Schiffrin EL: Peroxisome proliferator-activated receptors and cardiovascular remodeling. Am J Physiol Heart Circ Physiol 288: H1037-H1043, 2005.

18. Hu W, Yu Q, Zhang J and Liu D: Rosiglitazone ameliorates diabetic nephropathy by reducing the expression of Chemerin and ChemR23 in the kidney of streptozotocin-induced diabetic rats. Inflammation 35: 1287-1293, 2012.

19. Bergwitz C and Jüppner H: Regulation of phosphate homeostasis by PTH, vitamin D, and FGF23. Annu Rev Med 61: 91-104, 2010. 
20. Pontoriero G, Cozzolino M, Locatelli F and Brancaccio D: CKD patients: the dilemma of serum PTH levels. Nephron Clin Pract 116: c263-c268, 2010.

21. Levin A, Djurdjev O, Beaulieu M and Er L: Variability and risk factors for kidney disease progression and death following attainment of stage $4 \mathrm{CKD}$ in a referred cohort. Am J Kidney Dis 52: 661-671, 2008.
22. Kincaid-Smith P, Fairley KF, Farish S, Best JD and Proietto J: Reduction of proteinuria by rosiglitazone in non-diabetic renal disease. Nephrology (Carlton) 13: 58-62, 2008

23. Betz B, Schneider R, Kress T, Schick MA, Wanner C and Sauvant C: Rosiglitazone affects nitric oxide synthases and improves renal outcome in a rat model of severe ischemia/reperfusion injury. PPAR Res 2012: 219319, 2012. 